\title{
Preparation and Evaluation of Physicochemical Properties of the Doxepin Mucoadhesive Gel
}

\author{
Majid Rajaee ${ }^{1}$, Amir Talachi ${ }^{1}$, Abbas Pardakhty ${ }^{1}$, Ehsan Mohajeri ${ }^{1}$, Negar Dehghannoudeh ${ }^{2}$, \\ Mohammadzaman Basir ${ }^{3}$, Gholamreza Dehghannoudeh ${ }^{1,{ }^{*}}$ and Moslem Lari Najafi ${ }^{3,{ }^{* *}}$ \\ ${ }^{1}$ Pharmaceutics Department, Faculty of Pharmacy, Kerman University of Medical Sciences, Kerman, Iran \\ ${ }^{2}$ Faculty of Arts and Science, University of Toronto, Toronto, Canada \\ ${ }^{3}$ Pharmaceutics Research Center, Institute of Neuropharmacology, Kerman University of Medical Sciences, Kerman, Iran \\ "Corresponding author: Pharmaceutics Department, Faculty of Pharmacy, Kerman University of Medical Sciences, Kerman, Iran. Email: ghr_dehghan@kmu.ac.ir \\ "*orresponding author: Pharmaceutics Research Center, Institute of Neuropharmacology, Kerman University of Medical Sciences, Kerman, Iran. Email: \\ drlarinajafi@gmail.com
}

Received 2018 January 30; Revised 2019 May 06; Accepted 2019 May 12.

\section{Abstract}

Background: Oral mucositis is a common debilitating complication of cancer chemotherapy and radiotherapy that can reduce the quality of patient's lives. Hence, treating this condition plays an important role in increasing the patient's tolerance.

objectives: Doxepin mucoadhesive gel is useful for treating oral mucosa inflammation caused by long-term effects of chemotherapy, which has low adverse effects.

Methods: Doxepin gel's formulation was prepared with various concentrations of poloxamer 407 and hydroxypropyl methylcellulose in deionized water. The prepared gels were evaluated for $\mathrm{pH}$, appearance, viscosity, spreadability, stability, and drug release.

Results: After providing gels containing doxepin, formulations 1, 2, 8, and 9 had low quality and, thus, were removed from the study. Based on qualitative evaluations, formulations 3 and 4 did not meet the criteria for mucoadhesive gel and were removed from the study. The best formulation contained $17 \% \mathrm{w} / \mathrm{w}$ poloxamer $407,10 \% \mathrm{w} / \mathrm{w}$ hydroxypropyl methylcellulose, and $5 \% \mathrm{w} / \mathrm{w}$ doxepin.

Conclusions: Suitable physicochemical properties of prepared doxepin mucoadhesive gel enable it to well cover inflamed and damaged oral mucosa. On the other hand, doxepin's slow release from formulation (8 hours) can increase therapeutic effects and reduce side effects, which can heal and soothe inflammations of the oral mucosa and be useful in cancer patient's treatment.

Keywords: Mucositis, Mucoadhesive, Doxepin, Poloxamer 407, Viscosity, Stability Study

\section{Background}

Chemotherapy is an important part of treating several cancers. However, it may cause complications such as damage to the mucosa of the mouth, which occurs in more $40 \%$ of the patients $(1,2)$. In addition to reduced nutrition intake and poor oral hygiene, these painful lesions increase the risk of topical and systemic infection, which in turn negatively affect the chemotherapy process (3). Hence, relieving the pain of these oral lesions would be a great help for reducing the side effects of chemotherapy and patient tolerance. The use of topical formulations of lidocaine, Benzocaine, and diphenhydramine relieve side effects of chemotherapy, both temporarily and in the long-term.

Due to immunosuppressive characteristics, caution should be taken when prescribing steroidal antiinflammatory drugs. The effectiveness of other drugs has not yet been proven (4). Therefore, there is an urgent need for a special formulation with higher efficacy and lower side effects to manage inflammation of the oral mucosa. Doxepin is a tricyclic antidepressant with central and peripheral effects. The central activity of doxepin is inhibiting the reuptake of norepinephrine and serotonin at nerve terminals, and its peripheral activity is related to adenosine receptors. Moreover, it is a strong inhibitor of histamine receptors $\mathrm{H}_{1}$ and $\mathrm{H}_{2}$ (5).

In addition to treating depression and anxiety, it can also be used topically to treat itching dermatitis, and neuropathy pain $(6,7)$. Clinical studies showed doxepin mouthwash could significantly reduce oral mucosa damage (7).

\section{Objectives}

Doxepin mucoadhesive gel can be used for treating inflammation of oral mucosa due to long-term side effects of chemotherapy, with low adverse effects. 


\section{Methods}

\subsection{Materials}

Doxepin hydrochloride was obtained from Darupakhsh pharmaceuticals co, Iran. Hydroxypropyl methylcellulose (HPMC) and poloxamer 407 were obtained from BASF Ludwigshafen, Germany. Other chemicals used were of analytical grade.

\subsection{Equipment}

Digital balance (Sartorius MD BA100), UV-Visible spectrophotometer (UV-3100A Shimadzu Corporation, Japan), pH meter (Metrohm827 PH Lab), Magnetic stirrer (Heidolph), Viscometer (Brookfield DV-, Brookfield Engineering Laboratories, Inc. USA), Tensiometer (krüss k100.Germany).

\subsection{Method}

\subsubsection{Gel Preparation}

At first, different formulations with concentrations of Poloxamer 407 and Hydroxypropyl methylcellulose were prepared in deionized water, then the formulations were inspected visually and physically properties (Table 1 ).

\begin{tabular}{lccc}
\hline Table 1. Selected Formulations for Doxepin Mucoadhesive Gel \\
\hline & \multicolumn{3}{c}{ Materials } \\
\cline { 2 - 4 } $\begin{array}{l}\text { Formulation } \\
\text { Number }\end{array}$ & $\begin{array}{c}\text { Poloxamer 407 } \\
(\% \mathbf{W} / \mathbf{V})\end{array}$ & HPMC (\%W/V) & Doxepin $(\% \mathbf{W} / \mathbf{V})$ \\
\hline $\mathbf{1}$ & 8 & 2 & 5 \\
\hline $\mathbf{2}$ & 8 & 5 & 5 \\
\hline $\mathbf{3}$ & 8 & 10 & 5 \\
\hline $\mathbf{4}$ & 17 & 2 & 5 \\
\hline $\mathbf{5}$ & 17 & 5 & 5 \\
\hline $\mathbf{6}$ & 17 & 10 & 5 \\
\hline 7 & 30 & 2 & 5 \\
\hline $\mathbf{8}$ & 30 & 5 & 5 \\
\hline $\mathbf{9}$ & 30 & 10 & 5 \\
\hline
\end{tabular}

The poloxamer gels were prepared using the cold preparation technique (8). Different concentrations of poloxamer 407 were dispersed in $30 \mathrm{ml}$ of deionized water and constantly stirred to obtain a clear solution. Afterward, the obtained gel was stored in a refrigerator at $4{ }^{\circ} \mathrm{C}$ for $24 \mathrm{~h}$ to ensure complete dissolution. Then, accurately weighted doxepin was dissolved in $60 \mathrm{ml}$ of deionized water, and hydroxyl propyl methylcellulose (HPMC) was added. Both of the above solutions were mixed at $40^{\circ} \mathrm{C}-45^{\circ} \mathrm{C}$ with continuous stirring for 10 hours until the uniform gel was formed and stored in a glass container at room temperature. Finally, doxepin content, bioadhesives, viscosity, and drug release from the mucus membrane were evaluated $(9,10)$.

\subsubsection{Evaluation of Gel Formulations}

The gels were examined for their physical properties by visual inspection of color, clarity, homogeneity, and phase separation $(11,12)$.

\subsubsection{Thumb Test}

The thumb test was carried out by placing a little of mucoadhesive gel between the thumb and the middle finger and kept for some time. The quality of adhesiveness was measured by how difficult it would be to separate fingers (13).

\subsection{4. $p H$}

The $\mathrm{pH}$ values of the prepared gels were checked by using a $\mathrm{pH}$ meter, which was calibrated before each use with standard buffer solutions at $\mathrm{pH}=4,7$, and 9 (12).

\subsubsection{Viscosity}

A viscometer (Brookfield digital viscometer DV II RVTDV-II USA) was used to measure the viscosities of the gels. The spindle (model LV) was rotated at $60 \mathrm{rpm}$ at room temperature. Then, $100 \mathrm{~g}$ of the gel was taken in a beaker, and the spindle was dipped in for about 5 minutes, and the viscosities of gels were read (14).

\subsubsection{Occlusivity Test}

Occlusivity is one of the most important properties of mucoadhesives. In order to evaluate occlusivity, a 10\% gelatin solution was prepared and spread to a container with $1 \mathrm{~cm}$ height. Then, the plastic cylinders (inner diameter $3 \mathrm{~cm}$ ) were placed inside the container to form a gelatin layer inside them, then cylinders were removed, and $2 \mathrm{~mL}$ distilled water was poured in the opposite side of the cylinders. The cylinders were closed and weighed. The cells were put in a desiccator in $25 \pm 1$, and changes in weight of the cylinder were studied overtime again in the same test that $300 \mathrm{mg} / \mathrm{cm}^{2}$ of doxepin mucoadhesive gel was spread on the gelatin layer, and the water evaporation speed was measured through studying changes in weight of cells. In this test, the gelatin layer creates a similar skin condition. By comparing the water evaporation kinetic in the two states mentioned above, the occlusivity was calculated using the following equation:

Occlusivity percent $=\frac{a A-a B}{a A} \times 100$

In which $\mathrm{aA}$ and $\mathrm{aB}$ are water evaporation line slopes overtime for control (without mucoadhesive gel) and sample with mucoadhesive gel (15). 


\subsubsection{Spreadability}

The spreadability of the gel formulations of 5,6 , and 7 was determined by pressing $0.5 \mathrm{~g}$ of each prepared formula (at room temperature) between two glass slides for about 3 minutes and measuring the diameter of the formed circle. Then, a $250 \mathrm{~g}$ weight was placed on the center of a glass plate, and after 3 minutes' expansion of the gel was calculated. This action was repeated with a $500 \mathrm{~g}$ weight. All measurements were carried out in triplicate, and the results were compared with the standard (16).

\subsubsection{Adhesiveness}

The adhesion strength was determined by the tensiometer tool (Tensiometer kruss k100. Germany), and the mucoadhesive gels were tested for adhesiveness properties using a modified method developed by Yong et al. (17)

\subsubsection{In Vitro Release and Diffusion Studies}

To investigate in vitro release and diffusion studies of prepared gels, Franz diffusion cells were used. These cells consist of donor and receptor chambers and between two chambers a piece of Guinea pig oral mucosal tissue was positioned. The area of diffusion was $3-4 \mathrm{~cm}^{2}$. The doxepin mucoadhesive gels were spread onto the membrane, then the plastic layer (to prevent drying gel) was placed on it. In the receptor chamber, a specified volume of phosphate buffer was used. In order to remove the diffusion layers and dispersion of the drug, phosphate buffer with a magnetic stirring bar was continuously stirred (rpm 600) at $37 \pm 1$ degrees Celsius. Aliquots of $5 \mathrm{~mL}$ of the phosphate buffer were withdrawn at $0.5,1,2,3,4,5,6,7$, and $8 \mathrm{~h}$ and were replaced with an equal volume of fresh medium to maintain a constant volume. The concentration of doxepin was determined by UV spectroscopy at the $\lambda$ max of $297 \mathrm{~nm}$. The amount of doxepin released at various intervals of time was calculated and plotted versus time. All the experiments were run in triplicate, and the results were expressed as mean values \pm S.D (18)

\subsubsection{Stability Study}

The stability of doxepin mucoadhesive gel under triple different conditions, as mentioned below, was investigated. Doxepin mucoadhesive gel formulations were maintained in sealed and turbid glass containers over a period of three months at room temperature $\left(25 \pm 1^{\circ} \mathrm{C}\right)$. Then, at specified intervals (two weeks, one month, and three months), appearance, physical properties by visual inspection and thumb test were qualitatively evaluated, and viscosity, the active ingredient, mocoadhesiveness, and $\mathrm{pH}$ were quantitatively evaluated.

The formulations were maintained at $-10^{\circ} \mathrm{C}$ for four weeks, and the adhesion, uniformity, and touchablility of particles were evaluated by the finger test. Then, the results were compared with prototypes.

The formulation was centrifuged at $5000 \mathrm{rpm}$ in four and forty-degree Celsius for one minute. The crystalline growth, leakage, shrinking, bleeding, and gritting was investigated by microscope. Then, the results were compared with the prototype.

\section{Results}

After providing doxepin gels, formulations 1, 2, 8, and 9 had low quality and were removed from the study. The results of thumb test (from - to +++ ) are shown in Table 2. Simple gel (standard) was issued to select the appropriate formulations. Based on the qualitative evaluation, formulations 3 and 4 did not have the required characteristics for mucoadhesive gel and, therefore, were removed from the study. Hence, adhesion, uniformity, lack of palpable, and particles of formulations 5, 6, and 7 were studied.

\subsection{Spreadability}

Spreadability of formulations 5, 6, and 7, as well as the control gel, was evaluated. Based on the results, the spreading of formulations 5 and 6, compared to the control gel, were in the range of standard semi-solid products. According to Table 2, formulation 6 was selected as the best formulation.

\subsection{Occlusivity}

The results of the occlusivity test are shown in Figure 1.

Occlusivity $=\frac{[(0.031)-(0.025)]}{0.031} \times 100=19.354$

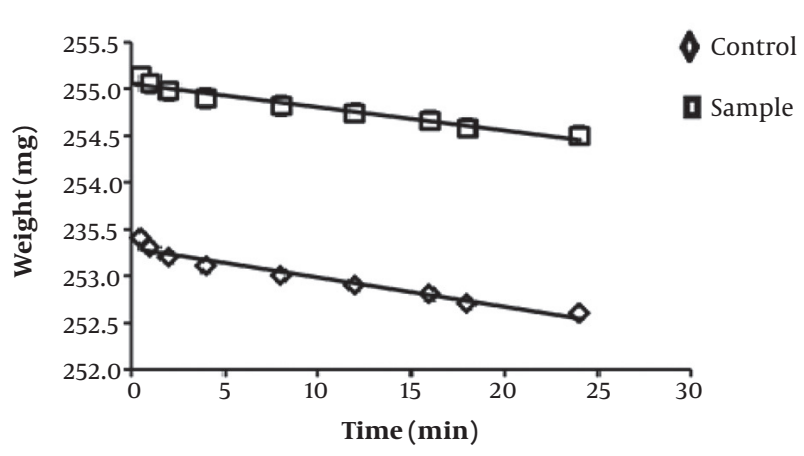

Figure 1. The weight of the control cells against time (temperature $25^{\circ} \mathrm{C}$ ). (Each of the values is measured three times. 


\begin{tabular}{|c|c|c|c|c|c|c|}
\hline Formulation Evaluation Items & Formulation 3 & Formulation 4 & Formulation 5 & Formulation 6 & Formulation 7 & Standard Gel \\
\hline Adherence & ++ & ++ & +++ & +++ & +++ & + \\
\hline Uniformity & ++ & + & +++ & +++ & +++ & +++ \\
\hline Lack of visible particles & ++ & +++ & +++ & +++ & +++ & +++ \\
\hline Physical properties & + & ++ & ++ & +++ & ++ & +++ \\
\hline
\end{tabular}

a +, Little; ++, medium; +++, a lot.

\subsection{Viscosity}

Rheological properties of formulation 6 were evaluated by viscometer at days $0,14,30$, and 90 and the results were $1298.23 \pm 0.24,1298.88 \pm 0.31,1399.47 \pm 0.53$, and $1300.31 \pm 0.42 \mathrm{cps}$, respectively (Figure 2 ).

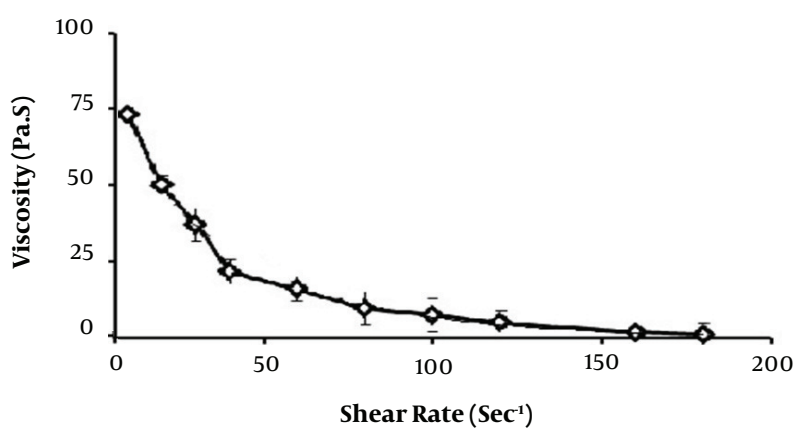

Figure 2. Viscosity formulation 6 versus increase shear rates (temperature $25^{\circ} \mathrm{C}$ ). (Each of the values is measured three times.)

\subsection{Adhesiveness}

The maximum mucoadhesiveness force of formulation 6 at special times was determined via measuring the surface tension by a tensiometer device.

\section{5. $p H$}

$\mathrm{pH}$ changes of formulation 6 were measured by the $\mathrm{pH}$ meter on a specific time period and changes in the results on days $0,14,30$, and 90 were in the range of $6.41 \pm 0.03$ to $6.22 \pm 0.04$.

In the final test, formulation 6 was placed into the centrifuge ( $5000 \mathrm{rpm}$ ) for one minute at 4 and $40^{\circ} \mathrm{C}$ temperatures, respectively, to compare with the prototype. According to the results, after centrifugation at $25^{\circ} \mathrm{C}$ crystal growth, fluid leak, shrinkage, and granules or fine particulate creation were not observed in the formulation. In the formulation which was centrifuged at $4^{\circ} \mathrm{C}$, fluid leakage and crystal growth happened.

\subsection{Diffusion and Release Drug}

Various models were tested for explaining the kinetics of drug release. To analyze the mechanism of the drug release rate and kinetics of the gel, the obtained data were fitted into zero-order and first-order, Higuchi, HixonCrowell model, and Korsmeyer-Peppas release model (Figure 3). The cumulative doxepin released from formulations 5, 6, and 7 were measured during an eight hours course, and kinetics of drug release was calculated.

\subsection{Stability Study}

The doxepin mucoadhessive gel was stored in a dark and sealed glass container for three months at room temperature $\left(25 \pm 1^{\circ} \mathrm{C}\right)$ and stored at specified intervals (2 weeks, 1 month, and 3 months) in terms of visual and physical properties. The finger test was quantitatively evaluated. Viscosity, amount of active ingredient, adhesive mucosa, and $\mathrm{pH}$ qualitatively were determined.

Appearance and physical properties, viscosity, uniformity, and adhesiveness at preparation time and 2 weeks, 1 month, and 2 months after gel preparations were almost constant, and there was no difference with the primary formulation. Formulation 6 was observed under a light microscope (magnification 100 times) when preparing and after one-month keeping at room temperature, which the formulation was uniformed.

\section{Discussion}

One of the major consequences of chemotherapy and radiotherapy is inflammation of oral mucosa, which has a significant effect on the quality of life of cancer patients. Hence, treating or preventing this problem is of crucial importance for cancer patients. Dhiman et al. (19) investigated the fluorouracil release of mucoadhesive formulations with different concentrations of poloxamer 407 , HPMC, and the mechanism of the fluorouracil release that follows Fick's law. In another similar study, Abdel-Mottaleb et al. (20) investigated the fluconazole release of mucoadhesive formulations with different concentrations of poloxamer 407, HPMC, and chitosan. They reported that 

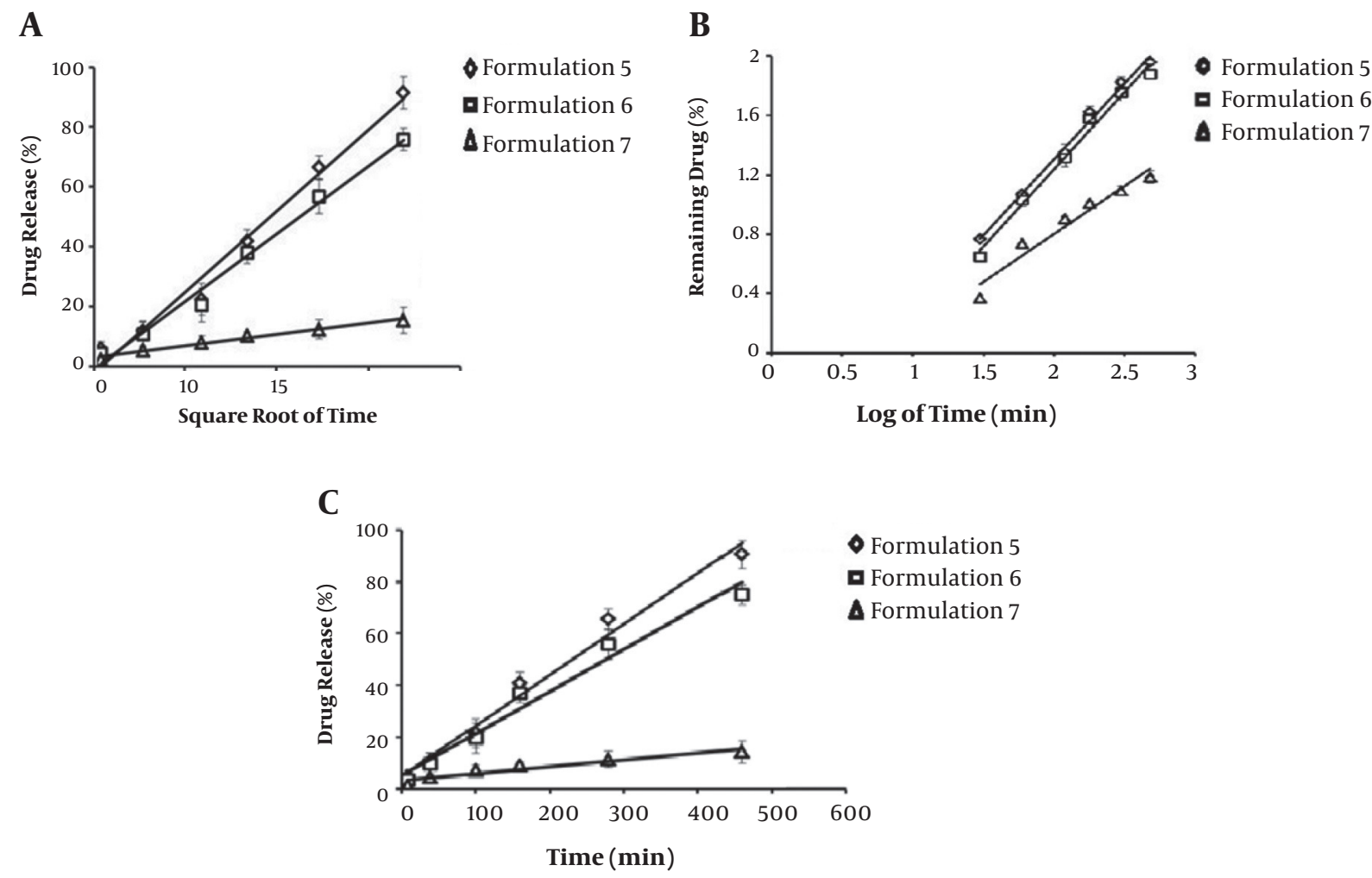

Figure 3. The order of release kinetic models of formulations 5, 6, and 7: A, Higuchi's kinetic model; B, Korsmeyer-Peppas model; and C, the zero-order kinetics model

the mechanism of drug release follows zero kinetics. In this study, the amount of drug released within 6 hours from the formulation with $20 \%$ poloxamer and $4 \%$ chitosan were $33 \%$ and $40 \%$, respectively (20). Another study by Perioli et al. (21) reported that metronidazole mucoadhesive gel with different concentrations (group I: 4\% hydroxyethyl cellulose (HEC) with 5/0,1, and $2 \%$ chitosan and group II: $4 \%$ hydroxyethylcellulose with 5/0, 1 and $2 \%$ of 5-methyl Pyrrolidinone chitosan) was developed for the treatment of bacterial vaginosis. In this study, the rate of drug release is obtained for 12 hours by Franz cells and cell membrane between $70 \%$ to $86 \%$ (21). In a study by Rossi et al. (22), benzydamine mucoadhesive gel hydrochloride was produced for the treatment of inflammation of the mucous from trimethyl chitosan and glycerol phosphate at a ratio of 1 to 2 for gel mucoadhesiveto treat inflammation of the mucous. They reported after 6 hours, about 70\% of the drug was released (22). According to the results, the release of doxepin from formulation 6 follows zero gradation, and the rheology study of formulation showed the plastic behavior of doxepin mucoadhesive gel. Another study by Baloglu et al. (23) reported that formulation viscosity was positively associated with temperature and con- centration of mucoadhesive polymers. The difference in viscosity depends on various factors, including physicochemical properties of the polymer, the concentration of polymer and drug, also the type of viscometer. As the concentration of polymers increases, more intermolecular bonds are formed in the polymer chains, which increases the viscosity of the formulation. Chemotherapy and radiotherapy also cause dry mouth (Xerostomia) (24). According to the literature, the application of mucoadhesive gel doxepin (19\%) may be beneficial to retain mucosa moisture. According to this study, it is expected that the mucoadhesive gel creates a protective barrier against external factors by covering the inflamed oral mucosa. On the other hand, by increasing the stay of the drug at the site of inflammation and releasing slowly doxepin creates a suitable anti-inflammatory effect and accelerates the healing of mucosal lesions.

Aksungur et al. (25) have used mucoadhesive polymer chitosan, as a protective covering against the candidate lesion. When separated as mucoadhesive tablets, they found no damage to the mouth tissue by removing the mucous lining. The results of surface tension detectors (Krüss K100), and power of mucoadhesive formulation 6 
via containing doxepin or lack of doxepin in the oral mucosa of pigs Hindi were $2.99 \pm 0.09$ dyne/cm and $5.04 \pm$ 0.03 dyne $/ \mathrm{cm}$, respectively, which do not indicate the impact of doxepin on the viscosity formulation. The amount of adhesion formulation 6 of doxepin was more desirable, compared to the simple skin gel (2.01 dyne/ $\left.\mathrm{cm}^{2}\right)$. Another study by Dhiman et al. (19) showed that the satisfying $\mathrm{pH}$ of different formulations was in the range of 5.5 to 7.2 , and they did not stimuli on the oral mucosa.

Changes of mucoadhesive gel formulation 6 doxepin, from the time of production until the end of the third month, was very low; also, the $\mathrm{pH}$ stayed in the range within the oral environment.

Meanwhile, mucoadhesive gel doxepin of physical characteristics within three months of qualitative finger test showed a slight decrease in viscosity, gel consistency, and physical durability during storage. Also, the quality of the doxepin mucoadhesive gel was estimated at an optimal level. Doxepin in mucoadhesive gel was also reduced to $97 \%$ at the end of three months and stayed in an acceptable range of $95 \%$ to $110 \%$. The adhesiveness of the doxepin mucoadhesive gel had a decreasing trend during a period of three months, but at the end of the third month, it was increased compared to the end of the first month. The decreased adhesion may be due to the loss of molecular bonds between polymers and polymers' destruction to monomers. By paying more attention to secondary links, the level of adhesion can be enhanced. When testing centrifuge at $4^{\circ} \mathrm{C}$, leakage and seepage of liquid Doxepin mucoadhesive gel were investigated, which can be attributed to the negative association between poloxamer viscosity and gel strength with temperature decrease. In the present study, the formulation of doxepin mucoadhesive gel was prepared, which has the ability to properly cover inflamed and damaged oral mucosa. This formulation has suitable physicochemical properties to cover the oral lesions, and because it releases the drug slowly (8 hours) and topically, it can increase therapeutic effect and reduce side effects of doxepin. It is hoped that doxepin mucoadhesive gel can be effective in caring for cancer patients by repairing and eliminating inflammation of the oral cavity.

\section{Acknowledgments}

We are thankful to Kerman University of Medical Sciences.

\section{Footnotes}

Authors' Contribution: Study design and supervisor: Gholamreza Dehghannoudeh, Abbas Pardakhty, and Ehsan
Mohajeri. Analysis and interpretation of data Majid Rajaee and Moslem Lari Najaf. Formulation, technical, and drafting of the manuscript: Amir Talachi, Negar Dehghannoudeh, and Mohammadzaman Basir.

Conflict of Interests: The authors declare no conflict of interest.

Funding/Support: The present research is financed by the Kerman University of Medical Sciences and this paper is a part of a pharmacy thesis at the Kerman University of Medical Sciences (Thesis No. 630).

\section{References}

1. Lionel D, Christophe L, Marc A, Jean-Luc C. Oral mucositis induced by anticancer treatments: physiopathology and treatments. Ther Clin Risk Manag. 2006;2(2):159-68. doi: 10.2147/tcrm.2006.2.2.159. [PubMed: 18360589]. [PubMed Central: PMC1661654].

2. Naidu MU, Ramana GV, Rani PU, Mohan IK, Suman A, Roy P. Chemotherapy-induced and/or radiation therapy-induced oral mucositis-complicating the treatment of cancer. Neoplasia. 2004;6(5):423-31. doi: 10.1593/neo.04169. [PubMed: 15548350]. [PubMed Central: PMC1531648].

3. Lalla RV, Sonis ST, Peterson DE. Management of oral mucositis in patients who have cancer. Dent Clin North Am. 2008;52(1):61-77. viii. doi: 10.1016/j.cden.2007.10.002. [PubMed: 18154865]. [PubMed Central: PMC2266835].

4. Hutchison TA, Shahan DR, Anderson ML. Drugdex System Internet version Micromedex Inc. Colorado: Greenwood Village; 2007.

5. Sweetman SC. Martindale: the complete drug reference. 3709. London: Pharmaceutical press; 2009.

6. Epstein JB, Epstein JD, Epstein MS, Oien H, Truelove EL. Oral doxepin rinse: the analgesic effect and duration of pain reduction in patients with oral mucositis due to cancer therapy. Anesth Analg. 2006;103(2):465-70. table of contents. doi: 10.1213/01.ane.0000223661.60471.78. [PubMed: 16861436].

7. Epstein JB, Epstein JD, Epstein MS, Oien H, Truelove EL. Doxepin rinse for management of mucositis pain in patients with cancer: one week follow-up of topical therapy. Spec Care Dentist. 2008;28(2):73-7. doi: 10.1111/j.1754-4505.2008.00015.x. [PubMed: 18402621].

8. Schmolka IR. Artificial skin. I. Preparation and properties of pluronic F-127 gels for treatment of burns. J Biomed Mater Res. 1972;6(6):571-82. doi: 10.1002/jbm.820060609. [PubMed: 4642986].

9. Ashour AE, Turnquist HR, Burns N, Wang X, Lin X, Tremayne J, et al. Flt3 ligand delivered in a pluronic formulation prolongs the survival of mice with orthotopic pancreatic adenocarcinoma. Cancer Biother Radiopharm. 2007;22(2):235-8. doi: 10.1089/cbr.2007.336. [PubMed: 17600470].

10. Bowen JM. Prevention and treatment of regimen-related mucosal toxicity. Recent Pat Anticancer Drug Discov. 2008;3(2):68-75. doi: 10.2174/157489208784638802. [PubMed: 18537748].

11. Shukr M, Metwally GF. Evaluation of Topical Gel Bases Formulated with Various Essential Oils for Antibacterial Activity against Methicillin- Resistant Staphylococcus Aureus. Trop J Pharm Res. 2014;12(6). doi: 10.4314/tjpr.v12i6.3.

12. Guleri TK. formulation and evaluation of topical gel of aceclofenac. $J$ Drug Delivery Ther. 2013;3(6). doi: 10.22270/jddt.v3i6.682.

13. Namdeo GR, Manohar SD, Bhanudas SR. World Journal of Pharmacy And Pharmaceutical Sciences. 2013.

14. Patel J, Patel B, Banwait H, Parmar K, Patel M. Formulation and evaluation of topical aceclofenac gel using different gelling agent. Int J Drug Dev Res. 2011;3(1):156-64. 
15. Derakhshandeh K, Abdollahipour R. Oralmucoadhesive paste of triamcinolone acetonide and zinc sulfate: Preparation and in vitro physicochemical characterization. J Rep Pharm Sci. 2014;3(2):115.

16. Soliman SM, Abdel Malak NS, El-Gazayerly ON, Abdel Rehim AA. Formulation of microemulsion gel systems for transdermal delivery of celecoxib: In vitro permeation, anti-inflammatory activity and skin irritation tests. Drug Discov Ther. 2010;4(6):459-71. [PubMed: 22491312].

17. Yong CS, Sah H, Jahng Y, Chang HW, Son JK, Lee SH, et al. Physicochemical characterization of diclofenac sodium-loaded poloxamer gel as a rectal delivery system with fast absorption. Drug Dev Ind Pharm. 2003;29(5):545-53. doi:10.1081/ddc-120018643. [PubMed: 12779284].

18. Salamat-Miller N, Chittchang M, Johnston TP. The use of mucoadhesive polymers in buccal drug delivery. Adv Drug Deliv Rev 2005;57(11):1666-91. doi: 10.1016/j.addr.2005.07.003. [PubMed 16183164].

19. Dhiman MK, Yedurkar PD, Sawant KK. Buccal bioadhesive delivery system of 5-fluorouracil: optimization and characterization. Drug Dev Ind Pharm. 2008;34(7):761-70. doi: 10.1080/03639040801926337. [PubMed: 18612914].

20. Abdel-Mottaleb MM, Mortada ND, Elshamy AA, Awad GA. Preparation and Evaluation of Fluconazole Gels. Egypt J Biomed Sci. 2007;23(1). doi: 10.4314/ejbs2.v23i1.40309.

21. Perioli L, Ambrogi V, Venezia L, Pagano C, Ricci M, Rossi C. Chitosan and a modified chitosan as agents to improve performances of mucoadhesive vaginal gels. Colloids Surf B Biointerfaces. 2008;66(1):141-5. doi: 10.1016/j.colsurfb.2008.06.005. [PubMed: 18621512].

22. Rossi S, Marciello M, Bonferoni MC, Ferrari F, Sandri G, Dacarro C, et al. Thermally sensitive gels based on chitosan derivatives for the treatment of oral mucositis. Eur J Pharm Biopharm. 2010;74(2):248-54. doi: 10.1016/j.ejpb.2009.10.003. [PubMed: 19854272].

23. Baloglu E, Karavana SY, Hyusein IY, Kose T. Design and formulation of mebeverine $\mathrm{HCl}$ semisolid formulations for intraorally administration. AAPS PharmSciTech. 2010;11(1):181-8. doi:10.1208/s12249-009-93743. [PubMed: 20101482]. [PubMed Central: PMC2850482].

24. Nicolatou-Galitis O. Oral Mucositis, Pain and Xerostomia in 135 Head and Neck Cancer Patients Receiving Radiotherapy with or without Chemotherapy. Open Cancer J. 2011;4(1):7-17. doi: $10.2174 / 1874079001104010007$.

25. Aksungur P, Sungur A, Unal S, Iskit AB, Squier CA, Senel S. Chitosan delivery systems for the treatment of oral mucositis: in vitro and in vivo studies. J Control Release. 2004;98(2):269-79. doi: 10.1016/j.jconrel.2004.05.002. [PubMed: 15262418]. 